\title{
Use of Nucleic Acid Amplification Testing for Diagnosis of Anorectal Sexually Transmitted Infections
}

\author{
Lisa A. Cosentino, ${ }^{\text {a,b }}$ Tracy Campbell, ${ }^{a}$ Abi Jett, ${ }^{a}$ Ingrid Macio, ${ }^{a, b}$ Tracy Zamborsky, ${ }^{a}$ Ross D. Cranston, ${ }^{d}$ and Sharon L. Hillier ${ }^{a, b, c}$ \\ Magee-Womens Research Institute, ${ }^{a}$ Microbicide Trials Network, ${ }^{b}$ and Departments of Obstetrics, Gynecology and Reproductive Sciences and Medicine, ${ }^{,}$Division of

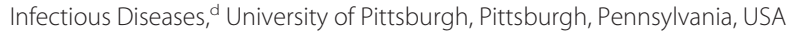

Nucleic acid amplification testing (NAAT) has become the preferred method to detect Chlamydia trachomatis and Neisseria gonorrhoeae, but no commercial tests are cleared by the U.S. Food and Drug Administration for use with rectal swab samples. This study evaluated the performance of strand displacement amplification (SDA) and transcription-mediated amplification (TMA) to detect C. trachomatis and N. gonorrhoeae and to determine if TMA could also detect Mycoplasma genitalium and Trichomonas vaginalis in men and women reporting a history of receptive anal intercourse. Discordant results between the NAATs were reevaluated using the Aptima CT or Aptima GC assay, each of which targets primers other than those targeted by the Aptima Combo 2 (AC2) assay, as the confirmatory test. Of 497 evaluable participants, 41 (8.2\%) were positive for C. trachomatis, 21 (4.2\%) were positive for N. gonorrhoeae, 26 (5.2\%) were positive for T. vaginalis, and 47 (9.5\%) were positive for M. genitalium. The sensitivity and specificity of the $C$. trachomatis test were $100 \%$ and $99.8 \%$ for AC2 and $56.1 \%$ and $100 \%$ for SDA, respectively. The sensitivity and specificity of the $N$. gonorrhoeae test were $100 \%$ and $100 \%$ for AC2 and $76.2 \%$ and $100 \%$ for SDA, respectively, while culture was only $23.8 \%$ sensitive. Of the 114 participants who had a positive result for any of the four infectious agents, 16 were positive for two pathogens and 3 were positive for three pathogens. These data suggest that rectal infection is common and that the AC2 is superior to SDA for the detection of C. trachomatis and N. gonorrhoeae from rectal swab samples.

$\mathrm{D}$ ue to its high sensitivity and specificity, nucleic acid amplification testing (NAAT) has become the preferred method for detection of Chlamydia trachomatis and Neisseria gonorrhoeae. NAAT detects and amplifies specific bacterial DNA or RNA sequences for the organism being tested and is highly sensitive and specific for the detection of C. trachomatis and N. gonorrhoeae by the use of swabs from the genital tract or of first-catch urine specimens $(8,19,22,28,30)$.

Anal intercourse is increasingly recognized as a component of the sexual repertoire of many couples. The Centers for Disease Control and Prevention (CDC) recommends screening for rectal sexually transmitted infections (STIs) for those reporting receptive anal intercourse (3). Kent et al. (14) reported that $53 \%$ of chlamydial infections and $64 \%$ of gonococcal infections were at nonurethral sites among men who have sex with men (MSM). However, since NAATs are not currently cleared for use with extragenital specimens by the U.S. Food and Drug Administration, verification is necessary to provide data to support the use of NAAT for rectal swab samples.

Several studies have shown that use of NAATs for detection of $N$. gonorrhoeae and C. trachomatis from rectal swab samples revealed a higher burden of disease than would have been detected with traditional assays, including culture $(21,26)$. In one large study that included 1,110 MSM, twice as many N. gonorrhoeae and C. trachomatis infections were detected by AC2 as by culture (26). There are limited published data on evaluation of rectal swabs from women for $N$. gonorrhoeae and C. trachomatis, even though $20 \%$ of U.S. women aged 20 to 39 who were surveyed reported having had receptive anal intercourse in the past year (12). Bachmann et al. (2) included 99 women in their study evaluating NAAT for detection of N. gonorrhoeae and C. trachomatis from rectal swabs and reported that $13 \%$ had either N. gonorrhoeae or C. trachomatis detected from the rectal swab sample.
Trichomonas vaginalis infection is the most common nonviral sexually transmitted disease worldwide. Trichomoniasis is a significant cause of morbidity among infected patients and has been linked to adverse outcomes, including pelvic inflammatory disease $(24,25)$, acquisition of HIV $(20,29)$, and HIV (16) shedding. Clinical diagnosis of trichomoniasis in women is made by performing a wet-mount microscopic examination and visualizing motile trichomonads in vaginal fluid. The gold standard for diagnosis is culture, which can take up to 7 days for a final result. NAAT has greater sensitivity than culture for the detection of $T$. vaginalis in vaginal samples (13). Crucitti et al. (4) showed that the presence of $T$. vaginalis in the rectum was highly correlated with the detection of $T$. vaginalis in the vagina. However, limited data from men are available on the detection of $T$. vaginalis from rectal swabs by the use of NAAT, with only 1 of 500 samples testing positive for this pathogen (5).

Mycoplasma genitalium is an organism that is found in the genital tract but had been difficult to detect until PCR became available in 1991. This organism has been linked with pelvic inflammatory disease (9), cervicitis (6), and urethritis (7). However, little is known about this organism as a cause of proctitis. In a study of 500 men attending a sexually transmitted disease (STD) clinic, the prevalence of M. genitalium was 5.4\% (5). Lillis et al.

Received 18 January 2012 Returned for modification 12 February 2012 Accepted 29 March 2012

Published ahead of print 4 April 2012

Address correspondence to Sharon L. Hillier, slh6+@pitt.edu.

Copyright $\odot$ 2012, American Society for Microbiology. All Rights Reserved. doi:10.1128/JCM.00185-12 
(18) reported the prevalence of $M$. genitalium in rectal swabs from 400 females to be $4.3 \%$.

The objective of the present study was to compare the performance of strand displacement amplification (SDA) to that of transcription-mediated amplification (TMA) for detection of C. trachomatis from rectal swab samples obtained from both men and women and to assess the performance of these NAAT systems compared to that of culture for detection of rectal N. gonorrhoeae in men and women reporting a history of receptive anal intercourse. In addition, we sought to describe the prevalence of $M$. genitalium and T. vaginalis in rectal swab samples by the use of the TMA system stratified by sex.

\section{MATERIALS AND METHODS}

Rectal swabs were collected from 500 participants aged 18 to 64 years attending the Allegheny County Health Department, Magee-Womens Hospital of University of Pittsburgh Medical Center (UPMC), or the Pittsburgh AIDS Center for Treatment who reported having had at least one lifetime episode of receptive anal intercourse. Written informed consent approved by each institutional review board was obtained from all participants prior to initiation of study procedures. Participants were excluded if they had taken oral antibiotics in the past 7 days or used a rectal douche or other rectal product in the previous $24 \mathrm{~h}$. Participants were asked a series of questions about their age, ethnicity, and sexual activity. Because published reports have documented the acceptability and quality of self-obtained rectal swabs $(1,15,23,27)$, participants were given the option to have the rectal swabs collected by the clinician performing the routine exam or to self-collect the three rectal swabs.

Clinician-obtained swabs were inserted approximately 1.5 to $2 \mathrm{~cm}$ above the anal margin and rotated for a few seconds. Participants opting to self-collect were instructed to do the same. First, an Aptima Unisex Collection Swab (AC2 procedure) (Aptima Combo 2 package insert IN0037-04 Rev A; Gen-Probe Inc., San Diego, CA) was collected followed by a Culturette Direct (SDA procedure) (Probetec package insert 3300754/AA; Becton-Dickinson, Sparks, MD). The final specimen used for N. gonorrhoeae culture was collected using a BD Transporter Swab with Charcoal (Becton-Dickinson, Sparks, MD). Samples were transported to the laboratory within $24 \mathrm{~h}$. Once at the laboratory, specimens were processed as recommended in the package insert for each product.

For C. trachomatis, true positives were based on two positive molecular tests (AC2 and SDA). For N. gonorrhoeae, a sample was considered a true positive if it was positive by culture or by two positive molecular tests (SDA and AC2). In the case of results that were discordant between SDA and AC2, the Aptima CT or Aptima GC assay, each of which targets different nucleic acid sequences, was used as the confirmatory test.

The charcoal swab for the culture detection of N. gonorrhoeae was stored at ambient temperature and inoculated onto Modified Thayer Martin media (PML Microbiologicals, Wilsonville, OR) within $24 \mathrm{~h}$ of collection. Plates were placed in 5 to $7 \% \mathrm{CO}_{2}$ at $36^{\circ} \mathrm{C}$ for $48 \mathrm{~h}$ (17), after which they were examined for the presence of N. gonorrhoeae. Identification was based on Gram stain, oxidase test, and the GonoGen II (BectonDickinson, Sparks, MD) identification system.

The Aptima rectal swab was tested for $T$. vaginalis by the use of the Aptima TV analyte-specific reagents (Gen-Probe) and the same methodology used with other TMA assays (10). The cutoff for a positive reaction was 60,000 relative light units. All positives were confirmed using an alternate primer set designed by Gen-Probe.

The Aptima rectal swab was tested in the same manner for M. genitalium by the use of the research-use-only Aptima TMA assay (11). The cutoff for a positive reaction was 40,000 relative light units. Because there were no alternative primers available for $M$. genitalium, all tests yielding an initial positive result were repeated and samples yielding two positive results were considered true positives.

Data analyses were conducted with SPSS statistical software, release
TABLE 1 Comparison of male and female study participants reporting a history of receptive anal intercourse $(n=497)$

\begin{tabular}{|c|c|c|c|c|}
\hline \multirow[b]{2}{*}{ Characteristic } & \multicolumn{3}{|c|}{$\begin{array}{l}\text { No. or median no. of participants (\% } \\
\text { or range) }\end{array}$} & \multirow[b]{2}{*}{$P$ value } \\
\hline & $\begin{array}{l}\text { Total } \\
(n=497)\end{array}$ & $\begin{array}{l}\text { Males } \\
(n=225)\end{array}$ & $\begin{array}{l}\text { Females } \\
(n=272)\end{array}$ & \\
\hline Age (median [range]) & $29(18-64)$ & $40(18-63)$ & $27(18-64)$ & $<0.001$ \\
\hline$<20 \mathrm{yr}$ & $52(10.5)$ & $20(8.8)$ & $32(11.8)$ & \\
\hline $21-30 \mathrm{yr}$ & $219(44.1)$ & $63(28)$ & $156(57.4)$ & \\
\hline $31-40 \mathrm{yr}$ & $75(15.1)$ & $32(14.2)$ & $43(15.8)$ & \\
\hline$>40 \mathrm{yr}$ & $151(30.4)$ & $110(48.9)$ & $41(15.1)$ & \\
\hline Race & & & & $<0.001$ \\
\hline Black & $232(46.7)$ & $73(32.4)$ & $159(58.5)$ & \\
\hline White & 247 (49.7) & $146(64.9)$ & $101(37.1)$ & \\
\hline Other & $18(3.6)$ & $6(2.7)$ & $12(4.4)$ & \\
\hline Clinic & & & & $<0.001$ \\
\hline HIV & $134(27.0)$ & $125(55.6)$ & $9(3.3)$ & \\
\hline Health Department & $225(45.3)$ & $92(40.9)$ & $133(48.9)$ & \\
\hline Outpatient & $138(27.7)$ & $8(3.6)$ & $130(47.8)$ & \\
\hline Collection & & & & 0.08 \\
\hline Clinician collected & $180(36.2)$ & $91(40.4)$ & $89(32.7)$ & \\
\hline Self-collected & $317(63.8)$ & $134(59.6)$ & $183(67.3)$ & \\
\hline $\begin{array}{l}\text { Gender of current sexual } \\
\text { partner }\end{array}$ & & & & 0.96 \\
\hline Female & $6(1.2)$ & $3(1.3)$ & $3(1.1)$ & \\
\hline Male & $402(80.9)$ & $182(80.1)$ & $220(80.9)$ & \\
\hline Both & $88(17.7)$ & $39(17.3)$ & $49(18)$ & \\
\hline Refused to answer & $1(0.2)$ & $1(0.4)$ & 0 & \\
\hline Rectal symptom(s) & & & & 0.14 \\
\hline Yes & $53(10.7)$ & $29(12.9)$ & $24(8.8)$ & \\
\hline No & $444(89.3)$ & $196(87.1)$ & $248(91.2)$ & \\
\hline
\end{tabular}

17.0.1 (SPSS, Inc., Chicago, IL). $P$ values were calculated using chi-square or Fisher's exact test analyses.

\section{RESULTS}

A total of 500 participants were enrolled in the study between May 2009 and March 2010; of those, 497 participants had complete evaluable swab sample sets. Two participants were excluded because they enrolled twice, and one participant was excluded because she admitted that the self-obtained swab was taken from the vagina. Of the evaluable study population, there were 225 (45\%) men with a median age of 40 years (range, 18 to 63 years) and $64.9 \%$ of the men were of white ethnicity. Of the $272(55 \%)$ women, the median age was 27 (range, 18 to 64 years) and 58.5\% identified themselves as black (Table 1).

There were a total of 41 rectal samples which were positive for C. trachomatis infection by two NAATs (Table 2). There were 18 rectal swab samples which were negative for $C$. trachomatis by SDA but positive by AC2. All 18 of these samples were confirmed to be positive for C. trachomatis by the Aptima CT test. There was one false-positive C. trachomatis test for AC2 which was negative by SDA and the Aptima CT. For N. gonorrhoeae, culture was compared to SDA and AC2. There were 21 gonococcal infections detected, and only five were detected by culture cultivation, for a sensitivity of $23.8 \%$. SDA detected 16 of 21 N. gonorrhoeae infections; all were confirmed to be positive by the Aptima GC test. 
TABLE 2 SDA, TMA, and culture sensitivities and specificities for detection of $N$. gonorrhoeae and C. trachomatis by reference standards

\begin{tabular}{|c|c|c|c|c|c|c|}
\hline Standard & Test & No. infected & $\%$ Sensitivity & No. uninfected & $\%$ Specificity & $P$ value \\
\hline N. gonorrhoeae & & & & & & $<0.0001$ \\
\hline \multirow{2}{*}{$\begin{array}{l}\text { Infected if two of three comparator tests positive; results discordant } \\
\text { between SDA and TMA resolved by Aptima GC }\end{array}$} & SDA & 16 & 76.2 & 478 & 100 & \\
\hline & TMA & 21 & 100 & 478 & 100 & \\
\hline C. trachomatis & & & & & & $<0.0001$ \\
\hline $\begin{array}{l}\text { Infected if both SDA and TMA positive; discordant results resolved } \\
\text { by Aptima CT }\end{array}$ & SDA & 23 & 56.1 & 456 & 100 & \\
\hline
\end{tabular}

Rectal infections were common in this study population. Fifty $(22 \%)$ of the men had at least one infection, and 63 (23\%) women had at least one pathogen detected. Of the 113 participants who had a positive result for any of the four infectious agents, nine women and seven men were positive for two different pathogens and one woman and two men were positive for three of the four pathogens. Of 497 participants, 20 men and 21 women were positive for C. trachomatis, 14 men and 7 women were positive for $N$. gonorrhoeae, 2 men and 24 women were positive for T. vaginalis, and 25 men and 22 women were positive for M. genitalium.

The gender-specific distribution of pathogens is shown in Table 3. Rectal C. trachomatis and M. genitalium were equally common among men and women. $N$. gonorrhoeae was more than twice as frequent in men as in women (6.2\% versus $2.6 \%)$, while $T$. vaginalis was detected primarily from the rectal swabs from women $(8.8 \%$ versus $0.9 \%)$, although it is noteworthy that $T$. vaginalis was detected from the rectal swabs obtained from two men. All T. vaginalis infections were confirmed through the use of the second set of primers.

\section{DISCUSSION}

Anal intercourse is very common, and the availability of highquality tests for detection of STIs by the use of rectal swabs is therefore a critical component of health care. The results of this study suggest that rectal culture for $N$. gonorrhoeae cannot be recommended, as it detected fewer than one in four of the gonococcal infections present. In addition, the results suggest that the AC2 system has substantially greater sensitivity than SDA for detection of both N. gonorrhoeae and C. trachomatis from rectal swabs.

There were several limitations to this study. First, urethral, pharyngeal, urine, and cervical samples were not collected, so it is unknown whether sexually transmitted pathogens were present at other body sites. Both Kent et al. (14) and Mimiaga et al. (21) reported that rectal swabs detect greater numbers of chlamydial and gonococcal infections in men than either urine or urethral swab samples. Therefore, failure to obtain and test rectal swab samples can result in a missed opportunity to detect sexually transmitted infections. Second, samples for culture of N. gonorrhoeae were held for up to $24 \mathrm{~h}$ prior to processing, which may

TABLE 3 Prevalence of rectal STIs stratified by sex

\begin{tabular}{llll}
\hline Species & $\begin{array}{l}\text { No. }(\%) \text { of men } \\
(n=225)\end{array}$ & $\begin{array}{l}\text { No. }(\%) \text { of women } \\
(n=272)\end{array}$ & $P$ value \\
\hline C. trachomatis & $20(8.9)$ & $21(7.7)$ & 0.627 \\
N. gonorrhoeae & $14(6.2)$ & $7(2.6)$ & 0.046 \\
T. vaginalis & $2(0.9)$ & $24(8.8)$ & $<0.0001$ \\
M. genitalium & $25(11.1)$ & $22(8.1)$ & 0.352 \\
\hline
\end{tabular}

have resulted in loss of viability during transport. Since the culture was prepared from the third sequential swab sample taken, the low sensitivity could reflect a reduced quantity of sample on the swab. However, Schachter et al. (26) also reported that the sensitivity of analysis of $N$. gonorrhoeae culture from rectal swabs was less than $50 \%$. Culture for C. trachomatis was not included in the present study, although it has been reported to be substantially less sensitive than NAAT in two published studies of rectal swab samples $(2,23)$.

In this study, the Aptima sample was always collected before the swab sample tested by SDA. It is therefore possible that swab order could have been a factor contributing to the suboptimal performance of SDA. In Bachmann's study (2), the order of rectal swab collection was rotated at 3-month intervals, and the SDA system was similar to TMA in sensitivity for both N. gonorrhoeae and C. trachomatis. However, Moncada et al. (23) reported in a study of 907 rectal samples that TMA had substantially greater sensitivity than SDA for both N. gonorrhoeae (84\% versus $77 \%$ ) and C. trachomatis ( $82 \%$ versus $41 \%$ ). In that study, the sample was placed in M4 media and then divided into aliquots for use in the different test platforms, which removed the variable of swab order from the evaluation. An earlier study by the same group of investigators found that TMA was superior to SDA for detection of C. trachomatis and N. gonorrhoeae from rectal swabs obtained from MSM (26). The results from the present study are in agreement with those of Moncada et al., indicating that TMA has greater sensitivity and specificity for detection of C. trachomatis and $N$. gonorrhoeae in rectal swabs obtained from both men and women.

In the present study, participants were given the option of having a clinician obtain the rectal swab sample or obtaining the swab samples themselves following brief instructions. In our study population, 317 (64\%) of the 497 participants chose to self-collect their samples. Self-collection of rectal swabs were equally acceptable, since roughly equal proportions of men (60\%) and women (67\%) chose self-collection. Moncada et al. (23) reported that the self-collected rectal swab samples from MSM were valid specimens, yielding sensitivities for $N$. gonorrhoeae and C. trachomatis which were similar to those seen with clinician-collected rectal swabs. It is possible that some of the results showing rectal $T$. vaginalis detected in women were attributable to vaginal fluid contamination of the perianal surface. However, the detection of $T$. vaginalis from the rectal swabs obtained from two men, both swabs having been collected by a clinician, suggests that contamination cannot account for all of the rectal samples positive for $T$. vaginalis. Self-collected rectal swabs may be ideal for use in screening for sexually transmitted infections in MSM. Our results suggest that women reporting receptive anal intercourse do have high 
rates of rectal $N$. gonorrhoeae and C. trachomatis infections and should be offered screening as well.

In summary, the Gen-Probe AC2 assay had high sensitivity and specificity for detection of C. trachomatis and N. gonorrhoeae from rectal swabs. Culture detection of $N$. gonorrhoeae from rectal swabs was only $24 \%$ sensitive and cannot be recommended to detect gonococcal proctitis. The Gen-Probe Aptima platform has the advantage that four pathogens ( N. gonorrhoeae, C. trachomatis, M. genitalium, and T. vaginalis) may be detected from one sample, and the pilot data presented here suggest that M. genitalium is equally common in rectal samples taken from men and women.

\section{ACKNOWLEDGMENTS}

Gen-Probe provided the reagents for T. vaginalis and M. genitalium testing, but they were not involved in the design of the study, analysis, and interpretation of the data and preparation or approval of the manuscript.

The Microbicide Trials Network is funded by the National Institute of Allergy and Infectious Diseases (5UM1AI068633), with cofunding from the National Institute of Child Health and Human Development and the National Institute of Mental Health, all components of the U.S. National Institutes of Health. The project described was supported by the National Institutes of Health through grants UL1RR024153 and UL1TR000005.

\section{REFERENCES}

1. Alexander S, et al. 2008. Self-taken pharyngeal and rectal swabs are appropriate for the detection of Chlamydia trachomatis and Neisseria gonorrhoeae in asymptomatic men who have sex with men. Sex. Transm. Infect. 84:488-492.

2. Bachmann LH, et al. 2010. Nucleic acid amplification tests for diagnosis of Neisseria gonorrhoeae and Chlamydia trachomatis rectal infections. J. Clin. Microbiol. 48:1827-1832.

3. Centers for Disease Control and Prevention. 2010. Sexually transmitted diseases treatment guidelines, 2010. MMWR Morb. Mortal. Wkly. Rep. 59:1-109.

4. Crucitti T, et al. 2010. Trichomonas vaginalis is highly prevalent in adolescent girls, pregnant women, and commercial sex workers in Ndola, Zambia. Sex. Transm. Dis. 37:223-227.

5. Francis SC, et al. 2008. Prevalence of rectal Trichomonas vaginalis and Mycoplasma genitalium in male patients at the San Francisco STD Clinic, 2005-2006. Sex. Transm. Dis. 35:797-800.

6. Gaydos C, Maldeis NE, Hardick A, Hardick J, Quinn TC. 2009. Mycoplasma genitalium as a contributor to the multiple etiologies of cervicitis in women attending sexually transmitted disease clinics. Sex. Transm. Dis. 36:598-606.

7. Gaydos C, Maldeis NE, Hardick A, Hardick J, Quinn TC. 2009. Mycoplasma genitalium compared to chlamydia, gonorrhea and trichomonas as an etiologic agent of urethritis in men attending STD clinics. Sex. Transm. Infect. 85:438-440.

8. Gaydos CA, et al. 2003. Performance of the APTIMA Combo 2 assay for detection of Chlamydia trachomatis and Neisseria gonorrhoeae in female urine and endocervical swab specimens. J. Clin. Microbiol. 41:304-309.

9. Haggerty CL, Taylor BD. 2011. Mycoplasma genitalium: an emerging cause of pelvic inflammatory disease. Infect. Dis. Obstet. Gynecol. 2011: 959816.

10. Hardick A, Hardick J, Wood BJ, Gaydos C. 2006. Comparison between the Gen-Probe transcription-mediated amplification Trichomonas vaginalis research assay and real-time PCR for Trichomonas vaginalis detection using a Roche LightCycler instrument with female self-obtained vaginal swab samples and male urine samples. J. Clin. Microbiol. 44:4197-4199.

11. Hardick J, et al. 2006. Performance of the Gen-Probe transcriptionmediated [corrected] amplification research assay compared to that of a multitarget real-time PCR for Mycoplasma genitalium detection. J. Clin. Microbiol. 44:1236-1240.

12. Herbenick D, et al. 2010. Sexual behavior in the United States: result from a national probability sample of men and women ages 14-94. J. Sex. Med. 7(suppl. 5):255-265.

13. Huppert JS, et al. 2007. Rapid antigen testing compares favorably with transcription-mediated amplification assay for the detection of Trichomonas vaginalis in young women. Clin. Infect. Dis. 45:194-198.

14. Kent CK, et al. 2005. Prevalence of rectal, urethral and pharyngeal chlamydia and gonorrhea detected in 2 clinical settings among men who have sex with men: San Francisco, California, 2003. Clin. Infect. Dis. 41:67-74.

15. Kent CK, et al. 2005. Self-collected rectal swabs-a novel method for detecting rectal chlamydia and gonorrhea among men who have sex with men seeking HIV testing services, abstr 861. Abstr. Infect. Dis. Soc. Am., San Francisco, CA, 6-9 October 2005.

16. Kissinger $\mathrm{P}$, et al. 2009. Trichomonas vaginalis treatment reduces vaginal HIV-1 shedding. Sex. Transm. Dis. 36:11-16.

17. Knapp JS, Holmes KK. 1975. Disseminated gonococcal infections caused by Neisseria gonorrhoeae with unique nutritional requirements. J. Infect. Dis. 132:204-208.

18. Lillis RA, Nsuami MJ, Myers L, Martin DH. 2011. Utility of urine, vaginal, cervical, and rectal specimens for detection of Mycoplasma genitalium in women. J. Clin. Microbiol. 49:1990-1992.

19. Martin DH, et al. 2000. Multicenter evaluation of AMPLICOR and automated COBAS AMPLICOR CT/NG tests for Neisseria gonorrhoeae. J. Clin. Microbiol. 38:3544-3549.

20. McClelland RS, et al. 2007. Infection with Trichomonas vaginalis increases the risk of HIV-1 acquisition. J. Infect. Dis. 195:698-702.

21. Mimiaga MJ, et al. 2008. Asymptomatic gonorrhea and chlamydial infections detected by nucleic acid amplification tests among Boston area men who have sex with men. Sex. Transm. Dis. 35:495-498.

22. Moncada J, et al. 2004. The effect of urine testing in evaluations of the sensitivity of the Gen-Probe APTIMA ${ }^{\circledR}$ Combo 2 assay on endocervical swabs for Chlamydia trachomatis and Neisseria gonorrhoeae: the infected patient standard reduces sensitivity of single site evaluation. Sex. Transm. Dis. 31:273-277.

23. Moncada J, Schachter J, Liska S, Shayevich C, Klausner JD. 2009. Evaluation of self-collected glans and rectal swabs from men who have sex with men for detection of Chlamydia trachomatis and Neisseria gonorrhoeae by use of nucleic acid amplification tests. J. Clin. Microbiol. 47: $1657-1662$.

24. Moodley P, Wilkinson D, Connolly C, Moodley J, Sturm AW. 2002. Trichomonas vaginalis is associated with pelvic inflammatory disease in women infected with human immunodeficiency virus. Clin. Infect. Dis. 34:519-522.

25. Paisarntantiwong R, et al. 1995. The relationship of vaginal trichomoniasis and pelvic inflammatory disease among women colonized with Chlamydia trachomatis. Sex. Transm. Dis. 22:344-347.

26. Schachter J, Moncada J, Liska S, Shayevich C, Klausner JD. 2008. Nucleic acid amplification tests in the diagnosis of chlamydial and gonococcal infections of the oropharynx and rectum in men who have sex with men. Sex. Transm. Dis. 35:637-642.

27. van der Helm JJ, et al. 2009. High performance and acceptability of self-collected rectal swabs for diagnosis of Chlamydia trachomatis and Neisseria gonorrhoeae in men who have sex with men and women. Sex. Transm. Dis. 36:493-497.

28. Van Der Pol B, et al. 2001. Multicenter evaluation of the BDProbeTec ET system for detection of Chlamydia trachomatis and Neisseria gonorrhoeae in urine specimens, female endocervical swabs and male urethral swabs. J. Clin. Microbiol. 39:1008-1016.

29. Van Der Pol B, et al. 2008. Trichomonas vaginalis infection and human immunodeficiency virus acquisition in African women. J. Infect. Dis. 197: $548-554$.

30. Van Der Pol B, et al. 2000. Multicenter evaluation of the AMPLICOR and automated COBAS AMPLICOR CT/NG tests for detection of Chlamydia trachomatis. J. Clin. Microbiol. 38:1105-1112. 